\title{
GINA recommendations in adults with symptomatic mild asthma and a smoking history
}

\author{
To the Editor:
}

The Global Initiative for Asthma (GINA) 2019 report makes a major change in asthma management by recommending that adults and adolescents with mild symptomatic asthma (Step 1) receive as-needed low-dose inhaled corticosteroid (ICS)-formoterol rather than a short-acting $\beta_{2}$-agonist alone [1]. At step 2, daily low-dose ICS is recommended, and GINA 2019 considers low-dose ICS-formoterol reliever as an alternative "preferred controller". The main evidence supporting these recommendations is provided by two large randomised controlled trials of inhaled budesonide-formoterol as-needed in mild asthma (SYGMA 1 and 2) [2,3] and from two "real-life" open label randomised controlled trials (Novel START and PRACTICAL) $[4,5]$. The strategy is considered generalisable across the spectrum of mild to moderate asthma, based on consistent findings from analysis of subgroups related to age, gender, smoking status and other factors [5].

How good is the evidence for the effectiveness of the low-dose ICS-formoterol reliever strategy in current and former smokers with mild asthma? There are several reasons to believe that further studies are required. Firstly, the proportion of participants with a smoking history recruited to low-dose budesonideformoterol reliever trials is much lower than the general population. In the SYGMA 1 and 2 studies, $<3 \%$ and $\leqslant 13 \%$ were current and former smokers, respectively, and in the Novel START and PRACTICAL trials, $<8 \%$ and $\leqslant 25 \%$ were current and former smokers, respectively. The prevalence rates for cigarette smoking amongst the 300 million people in the world with asthma match those in the general population of many countries $[6,7]$. In some high-income countries almost one quarter of the population are current smokers and a further quarter are former smokers. Several low-income and middle-income countries are at risk of worsening tobacco epidemics [8]. Secondly, participants with a smoking history enrolled in the trials had a low pack-year history, indicating a low intensity and short duration of exposure to tobacco smoke. The SYGMA 1 and 2 studies do not report the pack-year history of participants, but this is likely to be low, since smokers with a smoking history of $\geqslant 10$ pack-years were excluded $[2,3]$. The Novel START and PRACTICAL trials used less stringent exclusion criteria relating to smoking status: self-reported $>20$ pack-years smoking history, or the onset of respiratory symptoms after the age of 40 years in current or ex-smokers with $\geqslant 10$ pack-years smoking history $[4,5]$. Despite these more relaxed criteria, the median pack-year history in the Novel START treatment groups was only around 3 (interquartile range $0.5-9$ ) and in the PRACTICAL study groups was around 3 (1-8). Thirdly, the doses of budesonide administered in the budesonide-formoterol reliever strategy trials were $17 \%$ [2] to 50\% [5] lower than budesonide-formoterol (budesonide $400 \mu \mathrm{g}$ daily) maintenance treatment groups. Current smokers with mild to moderate asthma have impaired short-term responses to ICS compared to never smokers with asthma and may require higher doses of ICS to overcome corticosteroid insensitivity [7, 9, 10]. The dose of ICS used in the budesonide-formoterol reliever strategy trials may be insufficient to improve clinical outcomes in smokers with asthma, particularly in individuals with a higher intensity and longer duration of exposure to cigarette smoke ( $>10$ pack-year history).

The influence of exposure to tobacco smoke on the clinical features, immunopathology and response to treatment of asthma is complex and poorly understood [7]. Smokers with asthma have worse asthma control, more exacerbations and a greater reduction in lung function over time than non-smokers with

$@$ ERSpublications

The GINA recommendation to use as-required ICS-formoterol in symptomatic mild asthma is applicable to smokers with a low cumulative smoking history, but evidence is lacking for its effectiveness in smokers with medium and high tobacco use http://bit.ly/2Xqqluu

Cite this article as: Thomson NC, Chaudhuri R. GINA recommendations in adults with symptomatic mild asthma and a smoking history. Eur Respir J 2020; 55: 1902043 [https://doi.org/10.1183/ 13993003.02043-2019]. 
TABLE 1 Smoking and asthma associated factors to consider when assessing the generalisability of results of clinical trials of therapies in patients with asthma and a smoking history

\section{Smoking associated factors}

\section{Asthma associated factors}

- Smoking status

Current smoker

Former smoker

- Smoking exposure variables

Duration (years of smoking)

Intensity (cigarettes smoked daily)

Cumulative dose (pack-years): low $<10$ pack-years; medium $\geqslant 10$ to $\leqslant 20$ pack-years; high >20 pack-years

- Severity of asthma

Mild

Moderate

Severe

- Airway inflammatory phenotype/endotype

- Presence or absence of chronic airflow obstruction (FEV $1 / F V C<0.7$ ) (asthma-COPD overlap)

Other factors: Examples include demographic, genotypic, environmental exposures, adherence

$\mathrm{FEV}_{1}$ : forced expiratory volume in $1 \mathrm{~s}$; FVC: forced vital capacity.

asthma [7]. The most effective drug therapies for many asthma patients with a smoking history are uncertain, since most asthma trials exclude current or former smokers with $>10$ pack-year history due to concerns about including patients with COPD. Both smoking and asthma associated factors influence the response to drug therapies in asthma patients with a history of smoking, including differences between current smokers and former smokers and between individuals with a high intensity and long duration of exposure to tobacco smoke and those with a low cumulative smoking history (table 1).

In conclusion, the new GINA report recommendation on the use of as-required ICS-long-acting $\beta_{2}$-agonist in symptomatic mild or moderate asthma is applicable to current or former smokers with a low cumulative smoking history, but evidence is lacking for its effectiveness in current or former smokers with medium and high tobacco use, as is also the case for most other asthma treatment recommendations. Large "real-life" clinical trials of ICS-formoterol reliever therapy are required in moderate and heavy smokers with asthma and studies should include participants from low- and medium-income countries where the prevalence of cigarette smoking is often high. These studies will provide an evidence base for recommendations on drug treatment for the different subgroups of symptomatic current and former smokers with asthma.

Neil C. Thomson and Rekha Chaudhuri

Institute of Infection, Immunity and Inflammation, University of Glasgow, Glasgow, UK.

Correspondence: Neil C. Thomson, Institute of Infection, Immunity and Inflammation, University of Glasgow, Glasgow, G12 OYN, UK. E- mail: neil.thomson@glasgow.ac.uk

Received: 17 Oct 2019 | Accepted after revision: 13 Nov 2019

Author contributions: Conception, drafting the manuscript for important intellectual content, manuscript revision and final approval: all authors.

Conflict of interest: None declared.

\section{References}

1 Reddel HK, FitzGerald JM, Bateman ED, et al. GINA 2019: a fundamental change in asthma management. Eur Respir J 2019; 53: 1901046.

2 O'Byrne PM, FitzGerald JM, Bateman ED, et al. Inhaled combined budesonide-formoterol as needed in mild asthma. N Engl J Med 2018; 378: 1865-1876.

3 Bateman ED, Reddel HK, O’Byrne PM, et al. As-needed budesonide-formoterol versus maintenance budesonide in mild asthma. N Engl J Med 2018; 378: 1877-1887.

4 Beasley R, Holliday M, Reddel HK, et al. Controlled trial of budesonide-formoterol as needed for mild asthma. N Engl J Med 2019; 380: 2020-2030.

5 Hardy J, Baggott C, Fingleton J, et al. Budesonide-formoterol reliever therapy versus maintenance budesonide plus terbutaline reliever therapy in adults with mild to moderate asthma (PRACTICAL): a 52-week, open-label, multicentre, superiority, randomised controlled trial. Lancet 2019; 394: 919-928. 
6 To T, Stanojevic S, Moores G, et al. Global asthma prevalence in adults: findings from the cross-sectional world health survey. BMC Public Health 2012; 12: 204.

7 Thomson NC. Asthma and smoking-induced airway disease without spirometric COPD. Eur Respir J 2017; 49: 1602061.

8 Bilano V, Gilmour S, Moffiet T, et al. Global trends and projections for tobacco use, 1990-2025: an analysis of smoking indicators from the WHO Comprehensive Information Systems for Tobacco Control. Lancet 2015; 385: 966-976.

9 Tomlinson JEM, McMahon AD, Chaudhuri R, et al. Efficacy of low and high dose inhaled corticosteroid in smokers versus non-smokers with mild asthma. Thorax 2005; 60: 282-287.

10 van Schayck OCP, Haughney J, Aubier M, et al. Do asthmatic smokers benefit as much as non-smokers on budesonide/formoterol maintenance and reliever therapy? Results of an open label study. Respir Med 2012; 106: $189-196$.

Copyright @eERS 2020

From the author:

N.C. Thomson and R. Chaudhuri raise an important issue about treatment recommendations for patients with asthma who have a significant smoking history. The exclusion of such patients from most asthma studies appears to have largely been driven by the requirement by regulators for pure "asthma" and "COPD" populations in pharmacotherapy studies that are designed for obtaining regulatory approval. However, once that is obtained, there is an important need for additional clinical trials to be performed in broader populations, and with more pragmatic designs, to increase the generalisability of evidence to clinical practice. Considering the large number of randomised controlled trials in asthma in the past 20 years, very few studies have included patients with a significant smoking history; N.C. Thomson and R. Chaudhuri are to be commended for their work in this area.

The specific case raised by the authors relates to new recommendations made by the Global Initiative for Asthma (GINA) in 2019 [1]. The most fundamental of these changes is that, for adults and adolescents, treatment of asthma with short-acting $\beta_{2}$-agonists (SABA) alone is no longer recommended [2]. This change was possible because of evidence from four randomised controlled trials now totalling more than 10000 patients, demonstrating the efficacy and safety of as-needed low dose budesonide-formoterol in mild asthma. This regimen provided a $60-64 \%$ reduction in severe exacerbations compared with SABA alone $[3,4]$, and provided similar or greater risk reduction as with maintenance inhaled corticosteroids (ICS) [3-6], thus providing an efficacious alternative option to daily treatment in mild asthma. Given the need for regulatory approval of this regimen (already obtained in several countries) the initial two studies $[3,5]$ were limited to "pure" asthma populations, but as N.C. Thomson and R. Chaudhuri note, the subsequent two open-label studies $[4,6]$ allowed a greater smoking history. Of importance, interaction analyses in the latter two studies found no significant interaction between baseline smoking status and differential treatment response for exacerbations, severe exacerbations or symptom control. However, it would be highly desirable for additional studies to be done in mild asthma populations with larger numbers of smokers/ex-smokers and greater tobacco exposure, to give greater discriminatory power for such analyses.

Should GINA have excluded smokers or ex-smokers with a significant smoking history from its new recommendations until randomised controlled trials were available in these specific populations? I believe that the answer is no, because the primary recommendation by GINA was against SABA-only treatment, the concerns about which are not limited to "pure" asthma. Large well-conducted observational studies have shown that patients with diagnoses of both COPD and asthma are significantly more likely to die or be hospitalised if they are initiated on maintenance treatment with bronchodilators alone compared with ICS and long-acting $\beta_{2}$-agonists [7,8]. With such "hard" safety signals, the risks of treating asthma patients without any ICS cannot be ignored, even though the specific underlying mechanisms for this risk are not yet known.

@ERSpublications

The 2019 GINA recommendations are supported by clinical trials in $>10000$ mild asthma patients. For any treatment decision, GINA recommends that clinicians should consider individual patient characteristics/risk factors as part of shared decision making. http://bit.ly/2ZqboJ2

Cite this article as: Reddel HK. GINA recommendations in adults with symptomatic mild asthma and a smoking history. Eur Respir J 2020; 55: 2000068 [https://doi.org/10.1183/13993003.00068-2020]. 
What is the clinician to do, therefore, when a new asthma treatment becomes available, but no specific data are yet available for patient subgroups that may potentially have different responses? Since 2014, GINA has advocated against a one-size-fits-all approach to asthma management. Instead, GINA recommends that for treatment decisions in individual patients, the clinician should take into account not only population-level recommendations about efficacy, effectiveness and safety, but also the individual patient's characteristics or phenotype, noting as examples: "Does the patient have any features that predict differences in their future risk or treatment response compared with other patients (e.g. smoker; history of exacerbations, blood eosinophilia)? Are there any modifiable risk factors or comorbidities that may affect outcomes?" (box 3-3 of the GINA 2019 report [1]). The patient's preferences, and practical issues such as inhaler technique, likely adherence and treatment cost, may also form part of this shared decision-making [1]. Further, once treatment is started, patients who are at higher risk, such as smokers, should be followed up more closely to ensure that their treatment is appropriate. Meantime, for greater precision in treatment, we await the results of studies such as NOVELTY [9] that are investigating the specific mechanisms underpinning the heterogeneity and overlap of "asthma" and "COPD".

\section{Helen K. Reddel (1)}

Woolcock Institute of Medical Research, University of Sydney, Sydney, Australia.

Correspondence: Helen K. Reddel, Woolcock Institute of Medical Research, The University of Sydney, NSW, Australia. E-mail: helen.reddel@sydney.edu.au

Received: 13 Jan 2020 | Accepted after revision: 13 Jan 2020

Conflict of interest: H.K. Reddel reports research grants and personal fees for data monitoring committee work, consultancy, advisory board work and providing education from AstraZeneca, research grants, personal fees for data monitoring committee work, consultancy, advisory board work and providing education and non-financial support (study medication) from GlaxoSmithKline, personal fees for data monitoring committee work from Merck, research grants and personal fees for data monitoring committee work, advisory board work and providing education from Novartis, personal fees for providing education from Teva and Mundipharma, personal fees for advisory board work and providing education from Boehringer Ingelheim, personal fees for advisory board work from Sanofi Genzyme, outside the submitted work; and is Chair of the GINA Science Committee.

\section{References}

1 Global Initiative for Asthma. Global strategy for asthma management and prevention. www.ginasthma.org. Date last updated: 2019. Date last accessed: January 1, 2020.

2 Reddel HK, FitzGerald JM, Bateman ED, et al. GINA 2019: a fundamental change in asthma management: Treatment of asthma with short-acting bronchodilators alone is no longer recommended for adults and adolescents. Eur Respir J 2019; 53: 1901046.

3 O’Byrne PM, FitzGerald JM, Bateman ED, et al. Inhaled combined budesonide-formoterol as needed in mild asthma. N Engl J Med 2018; 378: 1865-1876.

4 Beasley R, Holliday M, Reddel HK, et al. Controlled trial of budesonide-formoterol as needed for mild asthma. N Engl J Med 2019; 380: 2020-2030.

5 Bateman ED, Reddel HK, O'Byrne PM, et al. As-needed budesonide-formoterol versus maintenance budesonide in mild asthma. N Engl J Med 2018; 378: 1877-1887.

6 Hardy J, Baggott C, Fingleton J, et al. Budesonide-formoterol reliever therapy versus maintenance budesonide plus terbutaline reliever therapy in adults with mild to moderate asthma (PRACTICAL): a 52-week, open-label, multicentre, superiority, randomised controlled trial. Lancet 2019; 394: 919-928.

7 Gershon AS, Campitelli MA, Croxford R, et al. Combination long-acting $\beta$-agonists and inhaled corticosteroids compared with long-acting $\beta$-agonists alone in older adults with chronic obstructive pulmonary disease. JAMA 2014; 312: 1114-1121.

8 Kendzerska T, Aaron SD, To T, et al. Effectiveness and safety of inhaled corticosteroids in older individuals with chronic obstructive pulmonary disease and/or asthma. A population study. Ann Am Thorac Soc 2019; 16: $1252-1262$.

9 Reddel HK, Gerhardsson de Verdier M, Agusti A, et al. Prospective observational study in patients with obstructive lung disease: NOVELTY design. ERJ Open Res 2019; 5: 00036-2018. 